\title{
LA ESCALA DE SEXISMO AMBIVALENTE EN ESTUDIANTES DE DOS UNIVERSIDADES DE AREQUIPA
}

\section{Ambivalent Sexism Inventory among students from two universities at Arequipa}

\author{
Sandra Fernández*, Walter L. Arias Gallegos** y Massiel Alvarado*** \\ Universidad Nacional de San Agustín, Arequipa \\ Universidad Católica San Pablo, Arequipa
}

\begin{abstract}
Resumen
En este estudio se analizaron las propiedades psicométricas del Inventario de Sexismo Ambivalente, para lue go valorar sus resultados, tras ser aplicado a una muestra de 434 estudiantes universitarios de dos universidades de la ciudad de Arequipa. Los valores psicométricos indican que los ítems son homogéneos y que cuenta con validez de constructo y confiabilidad adecuados $(\alpha=0.742)$. Se reportaron diferencias significativas entre varones y mujeres, siendo los varones más sexistas que aquellas, sobre todo en el sexismo hostil y benévolo paternalista y diferencia de género. En cuanto a la universidad de procedencia, los estudiantes de la universidad pública fueron más sexistas, así como quienes estudian carreras de ingenierías.
\end{abstract}

Palabras clave: Sexismo ambivalente, sexismo hostil, sexismo benévolo, psicometría.

\begin{abstract}
In this study we analyze the psychometric properties of Ambivalent Sexism Inventory, so then value its results after the application in a simple of 434 students from two universities at Arequipa City. The psychometric values indicate that the items are homogeneous and the test count with construct validity and reliability $(\alpha=0.742)$. The results, also note significant differences between men and women, being the males who are more sexist than females, specially, in hostile sexism, benevolent paternalism sexism, and gender differentiation benevolent sexism. About the university, those students who come from public university are more sexism, so as those who study engineering.
\end{abstract}

Keywords: Ambivalent sexism, hostile sexism, benevolent sexism, psychometric.

\footnotetext{
* Psicóloga. Docente de la Universidad San Agustín, Arequipa. lenniss@gmail.com

** Psicólogo. Docente de la Universidad Católica San Pablo, Arequipa. warias@ucsp.edu.pe

*** Psicóloga. Docente de la Universidad San Agustín, Arequipa. massiap@gmail.com
} 


\section{INTRODUCCIÓN}

Los términos sexo y género suelen utilizarse indistintamente (Gilbert, 1999), pero adoptaremos los conceptos más utilizados en la disciplina. Por ejemplo, Beckwith (1994, citado por Baron, 2005) distingue ambas palabras. El sexo se define en términos biológicos y se basa en las diferencias anatómicas y fisiológicas que están determinadas genéticamente. El género se refiere a cada cosa asociada con el sexo de un individuo, lo que incluye roles, comportamientos, preferencias y demás atributos que definen lo que significan masculinidad y femineidad en una cultura dada.

Así, la identidad de género indica el sentido subjetivo de ser varón o mujer. En los primeros años de vida casi todos nos damos cuenta de que somos varones o mujeres, pero nada garantiza que esta identidad corresponda al sexo biológico, y hay quienes sienten gran confusión al intentar identificar su masculinidad o feminidad (Crooks y Baur, 2010).

El rol de género (llamado a veces, erróneamente, rol sexual) designa una serie de actitudes y conductas consideradas normales y apropiadas en una cultura para individuos de un sexo en particular. Los roles crean expectativas de ciertos comportamientos sexuales que la gente debe cumplir. A la conducta socialmente apropiada para un varón se le llama masculina, mientras que se identifica como femenina a la conducta apropiada para una mujer (Crooks y Baur, 2010).

Por ejemplo, en relación con el lado positivo de los estereotipos de género de las mujeres, a ellas se les considera amables, cuidadosas y simpáticas; el otro lado de la moneda comprende la indecisión, la pasividad y una sensibilidad exagerada. De manera similar, se asume que los varones tienen rasgos tanto positivos (son decididos, asertivos y activos) como negativos (se comportan de forma agresiva, insensible y arrogante). Nótese que el estereotipo de género de las mujeres pareciera ser en cierto modo más favorable que el de los hombres, sin embargo, a pesar de este hecho las mujeres se enfrentan a un problema fundamental: los rasgos que se supone poseen, en principio positivos, las hace ser vistas como menos indicadas para desempeñar altos cargos o posiciones con rangos elevados, a diferencia de los rasgos asumidos para los varones. En definitiva, esto constituye una importante barrera para el avance de las mujeres (Heilman, 1995).

A ello se suma, que son muchas veces víctimas de estos estereotipos en diversos contextos, siendo la etapa universitaria, donde estos comienzan a manifestarse de manera más pronunciada, pero no solo en el plano de la sexualidad, sino también con respecto a sus roles de género, que terminan por ubicarlas como blanco fácil de la discriminación (Mingo y Moreno, 2015). En tal sentido, la presente investigación pretende valorar el sexismo ambivalente en un grupo de estudiantes universitarios de la ciudad de Arequipa, tanto varones como mujeres, con la finalidad de valorar las propiedades psicométricas del Inventario de Sexismo Ambivalente de Glick y Fiske (1996) y de presentar algunas diferencias de la muestra evaluada, en función del sexo, la universidad de origen y la carrera que estudian.

\section{EL SEXISMO AMBIVALENTE Y SU MEDICIÓN}

La primera aportación al sexismo, fue la de Allport (1954) al definir el prejuicio como: "una actitud hostil hacia una persona que pertenece a un grupo, simplemente porque pertenece a ese grupo, suponiéndose, por lo tanto, que posee las cualidades objetables atribuidas al grupo" (p. 7). Sin embargo, en el sexismo, dentro de los prejuicios ha sido un caso muy particular, que se han manifestado de manera ambivalente (Glick, \& Fiske, 1996). Así, aunque el sexismo, ha sido tradicionalmente concebido, como un reflejo de la hostilidad hacia las mujeres, este punto de vista, deja de lado los sentimientos subjetivamente positivos hacia ellas, que a menudo, van de la mano con antipatía sexista.

Actualmente, el sexismo se define, como una actitud discriminatoria, dirigida a las personas en virtud de su pertenencia a un determinado sexo biológico, en función del cual, se asumen diferentes características y conductas, y es considerado, un constructo multidimensional que abarca dos tipos de actitudes sexistas: el sexismo hostil y el sexismo benévolo (Glick y Fiske, 1996). 
Otra definición de sexismo es la que plantean Expósito, Moya y Glick (1998) donde el sexismo se entiende como una actitud dirigida hacia las personas en virtud de su pertenencia a los grupos basados en el sexo biológico, hombres o mujeres. Conceptualmente, toda evaluación (en las dimensiones cognitiva, afectiva y conductual) que se haga de una persona atendiendo a la categoría sexual biológica a la que pertenece puede ser etiquetada como "sexista", tanto si es negativa como positiva, y tanto si se refiere al hombre como a la mujer.

En los últimos años, viene siendo habitual distinguir, al menos en los países occidentales, entre las formas manifiestas u hostiles de sexismo y el constituido por las nuevas formas. Por sexismo manifiesto (también denominado clásico, viejo, hostil o a la antigua usanza), se entiende una actitud de prejuicio o conducta discriminatoria basada en la supuesta inferioridad o diferencia de las mujeres como grupo (Barbera y Martínez-Benlloch, 2004). Estas viejas creencias sexistas influyen en los juicios, evaluaciones y comportamientos que realizamos, produciendo discriminación.

Las nuevas formas de ideología de género sexista, comparten apariencias más encubiertas y sutiles de expresión, que pasan más inadvertidas, y que se siguen caracterizando por un tratamiento desigual y perjudicial hacia las mujeres. Sin embargo, esto no ha supuesto la superación de la discriminación "real" en función del género, que afecta en gran medida a la población mundial, lo que explica el interés que ha generado en las últimas décadas (Lameiras, Rodriguez, Carrera, \& Faílde, 2009). Además, a diferencia de lo que ocurre en cualquier otra relación intergrupal, las relaciones entre sexos, se caracterizan, por una compleja relación de fuerzas convergentes (dependencia) y divergentes (independencia), lo que le confiere su singularidad, pero también dificulta su comprensión (Glick, \& Fiske, 1996).

Así, de manera tradicional, el sexismo comprende tres dimensiones, como son el paternalismo dominador, la diferenciación de género competitiva y la hostilidad heterosexual. Sin embargo, con el surgimiento de los estudios de género, han surgido nuevas formas de sexismo. En el paradigma de Goldberg, se intenta explicar la discriminación laboral hacia las mujeres, en base a los estereotipos de género, de manera que un mismo trabajo es valorado de manera más positiva cuando lo realiza un hombre que cuando lo realiza una mujer. Se señala que estos prejuicios son más patentes, cuando un trabajo en particular, es típicamente realizado por varones. Otra forma de sexismo, es la que propone Deaux, cuando señala que, si una mujer tiene éxito en el trabajo, es más probable que se atribuya a factores externos que a sus propias capacidades, mientras que lo opuesto ocurre con los varones (Expósito, Moya, \& Glick 1998).

Estos estereotipos, han sido valorados de manera negativa para las mujeres, pero hay estudios que señalan que también tienen un lado positivo, que le juega en contra a los varones (Moya, 1990). Lo cierto es que toda actitud prejuiciosa, debe ser condenada socialmente, pues está vinculada a una serie de aspectos negativos que denigran al ser humano, y dificultan su real valoración, obstaculizando su desarrollo pleno en los diversos escenarios de la convivencia social. De hecho, los niveles de violencia (sexual, doméstica, etc.) que sufren las mujeres, incluso en sociedades avanzadas y democráticas, no reflejan la igualdad de género.

Una de las concepciones novedosas sobre el sexismo es la del neosexismo, propuesto por Tougas, Brown, Beaton y Joly (1995), y dirigida sobre todo a la captación de las concepciones políticas (especialmente las de carácter sutil) sobre la igualdad de género. Estos autores, definen el neosexismo como la manifestación de un conflicto entre los valores igualitarios y los sentimientos residuales negativos hacia las mujeres; y se centran en la esfera organizacional, donde intentan captar las formas que el sexismo adopta ante la incorporación masiva de las mujeres al mundo laboral, político y social.

En tal sentido, aunque se valora el aporte laboral de las mujeres y se reconoce una sobrecarga de sus roles familiares, se hace muy poco o nada por solucionar esta situación. Esta ambivalencia (el sexismo no está bien, pero las demandas de las mujeres son excesivas) se refleja en las justificaciones que los varones usan para el mantener su situación de ventaja sobre las mujeres. De esta manera, el neosexismo está relacionado con el 
sexismo hostil más tradicional, pues ambos comparten la actitud negativa hacia las mujeres. Según Tougas et al. (1995), un antecedente importante del neosexismo en los varones puede ser la percepción de amenaza hacia sus intereses colectivos.

En tal sentido, Moya y Expósito (2001) han adaptado la Escala de Neosexismo en España, y han encontrado en líneas generales, los mismos resultados que Tougas y su equipo, excepto en un punto: mientras que Tougas y sus colaboradores encontraron que la percepción subjetiva de amenaza a los intereses grupales era lo que estaba detrás del neosexismo (Tougas et al., 1995), Moya y Expósito (2001) encontraron que eran las creencias neosexistas las que estaban detrás de la percepción de amenaza a los intereses colectivos de los varones.

Una segunda concepción del sexismo que está teniendo bastante aceptación es la del sexismo ambivalente (Glick y Fiske, 1996). Según estos autores, el sexismo es ambivalente porque está formado por dos conceptos claramente diferenciados (aunque relacionados): el sexismo hostil y sexismo benevolente. Mientras que el primero coincide básicamente con el sexismo tradicional, el sexismo benévolo se define como un conjunto de actitudes interrelacionadas hacia las mujeres que son sexistas en cuanto las considera, de forma estereotipada, limitadas a ciertos roles, pero que tiene un tono afectivo positivo y tiende a suscitar en quien lo manifiesta, conductas categorizadas como prosociales (Expósito et al., 1998).

A diferencia del concepto de neosexismo, que como vimos, está centrado en el campo social o laboral, la concepción del sexismo ambivalente no sólo se refiere a las relaciones de género en ese campo, sino también a las relaciones íntimas. De este modo, el sexismo ambivalente pretende captar las características de las relaciones entre varones y mujeres, donde las diferencias de poder coexisten con la atracción interpersonal. Tanto el sexismo benévolo como el hostil tienen sus raíces en las condiciones biológicas y sociales prácticamente universales que muestran que, por una parte, los hombres poseen el control estructural de las instituciones económicas, legales y políticas pero, por otra parte, la reproducción sexual proporciona a las mujeres poder, en la medida en que los hombres tienen que depender de ellas para criar a sus hijos y, para la satisfacción de sus necesidades emocionales y sexuales (Glick y Fiske, 1996).

Según Glick y Fiske (1996), este sexismo benévolo sigue siendo sexismo, a pesar de los sentimientos positivos que pueda tener el perceptor, porque descansa en la dominación tradicional del varón y tiene aspectos comunes con el sexismo hostil: las mujeres están mejor en ciertos roles y espacios y son más débiles. De hecho, el sexismo benévolo puede ser incluso más perjudicial que el hostil, pues puede utilizarse para compensar o legitimar el sexismo hostil. Los componentes del sexismo benévolo son: el paternalismo protector, la diferenciación de género complementaria y la intimidad heterosexual.

Socialmente hablando, tenemos que el sexismo hostil y el sexismo benevolente actúan como un sistema articulado de recompensas y castigos con la finalidad de que las mujeres sepan cuál es su posición en la sociedad (Rudman y Glick, 2001). Por ejemplo, en el estudio de Moya et al. (2002) se encontró que la concepción del sexismo ambivalente tiene validez transcultural y muestra relación con el nivel de desarrollo humano del país. De esta forma, las mujeres comparten más que los hombres las creencias de sexismo benevolente en las culturas y sociedades con mayor desigualdad de género, mientras que los hombres comparten más fuertemente que las mujeres las creencias sexistas hostiles en las naciones y culturas menos desarrolladas y de mayor desigualdad.

Ahora bien, el sexismo ambivalente se ha valorado a través del Inventario de Sexismo Ambivalente que crean Glick y Fiske (1996), para valorar sus supuestos teóricos. La investigación psicométrica sobre este instrumento empero, sin embargo, no siempre ha corroborado sus supuestos teóricos. En España, se ha desarrollado el Inventario de Sexismo Ambivalente para Adolescentes, sobre la base de los supuestos teóricos de Glick y Fiske (1996), encontrando que las dimensiones de sexismo hostil y sexismo benévolo tienen índices adecuados de consistencia interna (0.84 y 0.77 , respectivamente); asimismo, con el análisis factorial exploratorio se han encontrado cinco factores, ya que el último factor no responde a ninguna estructura de varianza prevista teóricamente, y solo 
explica el $7.25 \%$ de la varianza. Se reportó también que el sexismo hostil se relacionó con la masculinidad, y que en las mujeres el sexismo benévolo correlaciona con feminidad (Lemus, Castillo, Moya, Padilla y Ryan, 2008).

En otro estudio psicométrico, realizado en Chile, se reportó que el Inventario de Sexismo Ambivalente era válido y confiable, siendo los varones quienes tenía puntuaciones más altas en sexismo hostil y benévolo que las mujeres (Cárdenas, Lay, González, Calderón, \& Alegría, 2010).

En Brasil, se valoraron las propiedades psicométricas del Inventario de Sexismo Ambivalente en tres muestras diferentes que sumaban 707 sujetos con edades entre 13 y 60 años. El análisis factorial confirmatorio, verificó la existencia de las dos dimensiones básicas: sexismo hostil y sexismo benevolente (Formiga, 2011).

En México en cambio, se evaluó a 347 habitantes con el Cuestionario de Sexismo Ambivalente y se logró confirmar la estructura de seis factores con cargas factoriales adecuadas, y se encontró que las mujeres están más de acuerdo con la diferencia complementaria de género y la intimidad heterosexual, mientras que los varones están más de acuerdo con el sexismo hostil (Cruz, Zempoaltecalt, \& Correa, 2005). En Argentina, se aplicó el Inventario de Sexismo Ambivalente a 345 sujetos, reportando que el análisis factorial confirmatorio tenía buenos índices de ajuste con el modelo teórico, pero mientras los factores del sexismo benévolo se diferenciaron claramente, los factores del sexismo hostil, se agruparon en un solo factor que explicaba el $28.21 \%$ de la varianza total (Vaamonde, \& Omar, 2012).

En el Perú, casi no existen estudios previos sobre el sexismo ambivalente, sin embargo, un estudio previo, realizado por Rottenbacher (2010), se analizó el sexismo ambivalente y el paternalismo masculino en función de la ideología política en adultos jóvenes de la ciudad de Lima. Se reportó que el conservadurismo político se relaciona con el sexismo ambivalente. Por otro lado, el año 2015, se publicó un trabajo sobre el neosexismo y la autoconcepto en adolescentes peruanos, donde se reporta que, en 350 escolares de
Arequipa, el neosexismo se relaciona inversamente con el autoconcepto, y que los varones son más neosexistas que las mujeres (Chino y Zegarra-Valdivia, 2015). A parte de estos estudios, no hay más trabajos que se avoquen a nuestra variable de estudio, pues en el país, los estudios relativos a la sexualidad son muy escasos. De parte de los médicos, se suele investigar la prevención de infecciones de transmisión sexual y las conductas de riesgo en adolescentes (Chirinos, Brindis, Salazar, Bardales, \& Reátegui, 1999; Gotuzzo, Peinado, Tijero, Mayorca, \& Badajos, 2001), mientras que los psicólogos han realizado estudios sobre la homofobia, tanto en Lima (Caycho, 2010) como en Arequipa (Portilla, \& Vilches, 2007).

\section{MÉTODO}

\section{Muestra}

La selección de la muestra fue no probabilística intencional. La muestra estuvo constituida por 434 participantes de los cuales 177 fueron varones y 257 mujeres con un rango de edad de entre 18 y 30 años. Los estudiantes provienen de la Universidad Católica San Pablo $(\mathrm{N}=147)$ y la Universidad Nacional de San Agustín ( $\mathrm{N}=287)$. Asimismo, 311 estudiantes cursan estudios en carreras de ingenierías y 115 en carreras de ciencias sociales.

\section{Instrumentos}

El instrumento utilizado fue el Inventario de Sexismo Ambivalente, creado por Glick y Fiske (1996), adaptado al español por Espósito, Muya y Glick (1998). El test consta de 22 ítems con una escala de respuesta tipo Likert con cinco alternativas que van desde Totalmente falso (1) hasta Totalmente cierto (5). La prueba es aplicable desde los 18 años y tiene una duración aproximada de 10 a 20 minutos para su resolución. Se compone de cuatro dimensiones:

- Sexismo Hostil: 2, 4, 5, 7, 10, 11, 14, 15, 16, 18, y 21.

- Sexismo Benévolo (paternalismo): 3, 9, 17 y 20

- Sexismo Benévolo (diferenciación de género): 8,19 y 22

- Sexismo Benévolo (intimidad heterosexual): 1, 6,13 y 12 . 
Cuenta con un coeficiente de fiabilidad, obtenido medianteel método de consistencia interna con la prueba Alfa de Cronbach de 0.91. La correlación entre los factores Sexismo hostil y Sexismo Benevolente fueron de $0.66(\mathrm{p}=.000)$. Para su calificación, un puntaje de 0 a 22 indica bajo sexismo hostil y benevolente, de 23 a 39 , un sexismo moderado para ambas dimensiones, y de 40 a 45 un sexismo benevolente alto, y de 40 a 55 , un sexismo hostil alto.

\section{Procedimiento}

La aplicación del cuestionario se realizó de forma colectiva en las áreas de sociales e ingenierías de la Universidad Católica San Pablo y la Universidad Nacional de San Agustín, con una duración de 30 minutos y bajo la supervisión de un miembro del equipo de la investigación. Después de explicar el objetivo general del estudio, se hizo énfasis en el carácter voluntario y confidencial de la participación y de los datos brindados. Asimismo, les comunicó que la información sería utilizada solo para cumplir con los fines del estudio y si se sentían incómodos en algún momento, podían dejar de desarrollar el cuestionario sin que ello los perjudicara. Los directores de las universidades y los profesores facilitaron la evaluación de la muestra en horarios de clase.

\section{Resultados}

Para el caso de la valoración psicométrica del Inventario de Sexismo Ambivalente (ISA) tenemos que todos los ítems registran correlaciones ítem-test superiores al mínimo establecido de 0.20 (Livia y Ortiz, 2014) cuyo rango va de 0.401 a $0.672(\mathrm{p}<0.000)$, por lo que, todos los reactivos del instrumento, se muestran homogéneos y miden lo mismo (ver Tabla 1).
Tabla 1

Correlaciones ítem-test del ISA

\begin{tabular}{lccc}
\hline $\mathbf{N}^{\circ}$ de Ítem & r ítem-test & $\mathbf{N}^{\circ}$ de Ítem & r ítem-test \\
\hline 1 & 0.534 & 12 & 0.639 \\
2 & 0.510 & 13 & 0.586 \\
3 & 0.486 & 14 & 0.672 \\
4 & 0.507 & 15 & 0.611 \\
5 & 0.595 & 16 & 0.583 \\
6 & 0.526 & 17 & 0.472 \\
7 & 0.505 & 18 & 0.542 \\
8 & 0.401 & 19 & 0.449 \\
9 & 0.468 & 20 & 0.510 \\
10 & 0.641 & 21 & 0.493 \\
11 & 0.632 & 22 & 0.453 \\
\hline
\end{tabular}

Asimismo, se realizó un análisis factorial para valorar la validez de constructo de la prueba. El valor KMO obtenido es de 0.901 y la prueba de esfericidad de Bartlett arrojó un valor chi cuadrado de X2= $2956.25492(\mathrm{gl}=231)$ y un nivel de significancia de $\mathrm{p}<0.000$, por lo que la prueba puede someterse a factorización.

Tras el análisis factorial se obtuvieron cinco factores que explican el $55.342 \%$ de la varianza total del instrumento por lo que se concluye que la prueba es multidimensional, sin embargo, la versión original tiene cuatro factores. En nuestro caso el primer factor explica el $19.549 \%$ de la varianza total, el segundo factor explica el $12.487 \%$, el tercero explica el $8.462 \%$ de la varianza, el cuarto facto explica el $8.001 \%$ de la varianza total y el quinto factor, explica el $6.841 \%$.

$\mathrm{Al}$ hacer la rotación varimax se tiene que solo el ítem 9 satura en el quinto factor (ver Tabla 2), por lo que se decidió tomar la saturación inmediatamente más alta y ubicarlo en el factor 4 y así desaparecer el factor 5, por lo que la versión final del instrumento quedó constituida de los siguientes ítems:

Factor 1: Sexismo hostil $(2,4,5,7,10,11,14,15$, $16,18,21)$

Factor 2: Sexismo benevolente intimidad heterosexual $(1,6,12,13)$

Factor 3: Sexismo benevolente paternalismo (3, 17, 20, 22)

Factor 4: Sexismo benevolente diferenciación de género $(8,9,19)$ 
Tabla 2

Matriz de componentes rotados con método varimax ASI

\begin{tabular}{|c|c|c|c|c|c|}
\hline & 1 & 2 & 3 & 4 & 5 \\
\hline Ítem 01 & & 0.751 & & & \\
\hline Ítem 02 & 0.652 & & & & \\
\hline Ítem 03 & & & 0.406 & & \\
\hline Ítem 04 & 0.612 & & & & \\
\hline Ítem 05 & 0.580 & & & & \\
\hline Ítem 06 & & 0.679 & & & \\
\hline Ítem 07 & 0.513 & & & & \\
\hline Ítem 08 & & & & 0.703 & \\
\hline Ítem 09 & & & & 0.309 & 0.692 \\
\hline Ítem 10 & 0.581 & & & & \\
\hline Ítem 11 & 0.676 & & & & \\
\hline Ítem 12 & & 0.735 & & & \\
\hline Ítem 13 & & 0.775 & & & \\
\hline Ítem 14 & 0.655 & & & & \\
\hline Ítem 15 & 0.645 & & & & \\
\hline Ítem 16 & 0.708 & & & & \\
\hline Ítem 17 & & & 0.464 & & \\
\hline Ítem 18 & 0.562 & & & & \\
\hline Ítem 19 & & & & 0.721 & \\
\hline Ítem 20 & & & 0.750 & & \\
\hline Ítem 21 & 0.437 & & & & \\
\hline Ítem 22 & & & 0.613 & & \\
\hline
\end{tabular}

Asimismo, se realizó el análisis de la confiabilidad mediante el método de consistencia interna, para lo cual se aplicó la prueba Alfa de Cronbach, obteniéndose un

Tabla 3

Valores descriptivos del sexismo y la violencia expresada y percibida

\begin{tabular}{lrcrrr}
\hline & $\begin{array}{c}\text { Sexismo } \\
\text { hostil }\end{array}$ & $\begin{array}{c}\text { Sexismo B } \\
\text { heterosexual }\end{array}$ & $\begin{array}{c}\text { Sexismo B } \\
\text { paternalista }\end{array}$ & $\begin{array}{c}\text { Sexismo B } \\
\text { Diferenc. género }\end{array}$ & $\begin{array}{c}\text { Total } \\
\text { Sexismo }\end{array}$ \\
\hline Media & 30.615 & 10.594 & 14.073 & 6.324 & 61.608 \\
Mediana & 31 & 11 & 14 & 7 & 63 \\
Moda & 26 & 13 & 16 & 5 & 59 \\
Desv. típ. & 10.863 & 5.381 & 4.933 & 2.443 & 18.421 \\
Varianza & 118.010 & 28.962 & -0.185 & -0.714 & 339.333 \\
Asimetría & -0.319 & -0.232 & -0.274 & 0.096 & -0.505 \\
Curtosis & -0.378 & -0.761 & 0 & 0 & 0.015 \\
Mínimo & 0 & 0 & 25 & 10 & 0 \\
Máximo & 54 & 20 & & 99 \\
\hline
\end{tabular}

índice de 0.742 , lo que quiere decir que la prueba es confiable. 
Con respecto al análisis de los datos, los valores descriptivos nos permiten apreciar que en la Tabla 3, el valor de sexismo hostil es moderado, el del sexismo benévolo heterosexual es bajo, al igual que el del sexismo benévolo paternalista y el sexismo benévolo diferenciación de género; mientras que el sexismo como puntuación total es alto. La curtosis del sexismo hostil, el sexismo benevolente heterosexual y el sexismo benevolente paternalista también son mayores que el sexismo benévolo diferenciación de género.

Tabla 4

Comparaciones sexismo por género

\begin{tabular}{|c|c|c|c|c|c|c|c|}
\hline Variables & Sexo & $\mathbf{N}$ & Media & $\begin{array}{c}\text { Desviación } \\
\text { típ. }\end{array}$ & $\begin{array}{l}\text { Error típ. } \\
\text { de media }\end{array}$ & $\mathbf{t}$ & $\mathbf{p}$ \\
\hline \multirow[t]{2}{*}{ Sexismo hostil } & Varón & 177 & 35.288 & 10.729 & 0.806 & \multirow[t]{2}{*}{7.813} & \multirow[t]{2}{*}{0.000} \\
\hline & Mujer & 257 & 27.396 & 9.744 & 0.607 & & \\
\hline \multirow{2}{*}{$\begin{array}{l}\text { Sexismo benévolo } \\
\text { heterosexual }\end{array}$} & Varón & 177 & 11.169 & 5.319 & 0.399 & \multirow[t]{2}{*}{1.857} & \multirow[t]{2}{*}{0.046} \\
\hline & Mujer & 257 & 10.198 & 5.398 & 0.336 & & \\
\hline \multirow{2}{*}{$\begin{array}{l}\text { Sexismo benévolo } \\
\text { paternalista }\end{array}$} & Varón & 177 & 14.762 & 5.057 & 0.380 & \multirow[t]{2}{*}{2.404} & \multirow[t]{2}{*}{0.016} \\
\hline & Mujer & 257 & 13.599 & 4.798 & 0.299 & & \\
\hline \multirow{2}{*}{$\begin{array}{l}\text { Sexismo } \\
\text { dif. de género }\end{array}$} & Varón & 177 & 5.796 & 2.447 & 0.183 & \multirow[t]{2}{*}{-3.774} & \multirow[t]{2}{*}{0.000} \\
\hline & Mujer & 257 & 6.688 & 2.377 & 0.148 & & \\
\hline \multirow{2}{*}{$\begin{array}{l}\text { Sexismo } \\
\text { ambivalente }\end{array}$} & Varón & 177 & 67.016 & 18.564 & 1.395 & \multirow[t]{2}{*}{5.166} & \multirow[t]{2}{*}{0.000} \\
\hline & Mujer & 257 & 57.883 & 17.402 & 1.085 & & \\
\hline
\end{tabular}

En cuanto a las comparaciones por sexo, el sexismo hostil es mayor en los varones $(p=0.000)$, al igual que el sexismo benévolo heterosexual $(\mathrm{p}=0.046)$, el sexismo benévolo paternalista $(\mathrm{p}=0.016)$ y el sexismo ambivalente $(\mathrm{p}=0.000)$; pero el sexismo de diferencia de género es mayor en las mujeres $(\mathrm{p}=0.000)$.

Tabla 5

Comparaciones sexismo por carrera

\begin{tabular}{|c|c|c|c|c|c|c|c|}
\hline Variables & Carrera & $\mathbf{N}$ & Media & $\begin{array}{c}\text { Desviación } \\
\text { típ. }\end{array}$ & $\begin{array}{l}\text { Error típ. } \\
\text { de media }\end{array}$ & $\mathbf{t}$ & $\mathbf{p}$ \\
\hline Sexismo hostil & $\begin{array}{r}\text { Ingeniería } \\
\text { Sociales }\end{array}$ & $\begin{array}{l}309 \\
125\end{array}$ & $\begin{array}{l}30.860 \\
30.008\end{array}$ & $\begin{array}{l}10.795 \\
11.049\end{array}$ & $\begin{array}{l}0.614 \\
0.988\end{array}$ & 0.732 & 0.464 \\
\hline $\begin{array}{l}\text { Sexismo benévolo } \\
\text { heterosexual }\end{array}$ & $\begin{array}{r}\text { Ingeniería } \\
\text { Sociales }\end{array}$ & $\begin{array}{l}309 \\
125\end{array}$ & $\begin{array}{r}10.851 \\
9.960\end{array}$ & $\begin{array}{l}5.360 \\
5.403\end{array}$ & $\begin{array}{l}0.304 \\
0.483\end{array}$ & 1.559 & 0.120 \\
\hline $\begin{array}{l}\text { Sexismo benévolo } \\
\text { paternalista }\end{array}$ & $\begin{array}{r}\text { Ingeniería } \\
\text { Sociales }\end{array}$ & $\begin{array}{l}309 \\
125\end{array}$ & $\begin{array}{l}14.411 \\
13.240\end{array}$ & $\begin{array}{l}4.635 \\
5.534\end{array}$ & $\begin{array}{l}0.263 \\
0.495\end{array}$ & 2.087 & 0.038 \\
\hline $\begin{array}{l}\text { Sexismo dif. } \\
\text { de género }\end{array}$ & $\begin{array}{r}\text { Ingeniería } \\
\text { Sociales }\end{array}$ & $\begin{array}{l}309 \\
125\end{array}$ & $\begin{array}{l}6.446 \\
6.024\end{array}$ & $\begin{array}{l}2.279 \\
2.795\end{array}$ & $\begin{array}{l}0.129 \\
0.250\end{array}$ & 1.500 & 0.135 \\
\hline $\begin{array}{l}\text { Sexismo } \\
\text { ambivalente }\end{array}$ & $\begin{array}{r}\text { Ingeniería } \\
\text { Sociales }\end{array}$ & $\begin{array}{l}309 \\
125\end{array}$ & $\begin{array}{l}62.569 \\
59.232\end{array}$ & $\begin{array}{l}17.837 \\
19.662\end{array}$ & $\begin{array}{l}1.014 \\
1.758\end{array}$ & 1.643 & 0.101 \\
\hline
\end{tabular}

También se hicieron comparaciones de las puntuaciones obtenidas en sexismo ambivalente en función del área de estudios (sociales e ingenierías) (Tabla 5), encontrándose diferencias estadísticamente significativas en el sexismo benévolo paternalista, de modo que los estudiantes de ingenierías presentan puntajes mayores que los estudiantes de sociales $(\mathrm{t}=$ 2.087; $\mathrm{p}=0.038$ ). 
Tabla 6

Comparaciones de sexismo por universidad

\begin{tabular}{|c|c|c|c|c|c|c|c|}
\hline Variables & Universidad & $\mathbf{N}$ & Media & $\begin{array}{c}\text { Desviación } \\
\text { típ. }\end{array}$ & $\begin{array}{l}\text { Error típ. } \\
\text { de media }\end{array}$ & $\mathbf{t}$ & $\mathbf{p}$ \\
\hline Sexismo hostil & $\begin{array}{l}\text { UNSA } \\
\text { UCSP }\end{array}$ & $\begin{array}{l}287 \\
147\end{array}$ & $\begin{array}{l}31.501 \\
28.884\end{array}$ & $\begin{array}{l}10.106 \\
12.055\end{array}$ & $\begin{array}{l}0.596 \\
0.994\end{array}$ & 2.257 & 0.024 \\
\hline $\begin{array}{l}\text { Sexismo benévolo } \\
\text { heterosexual }\end{array}$ & $\begin{array}{l}\text { UNSA } \\
\text { UCSP }\end{array}$ & $\begin{array}{l}287 \\
147\end{array}$ & $\begin{array}{l}10.787 \\
10.217\end{array}$ & $\begin{array}{l}5.159 \\
5.790\end{array}$ & $\begin{array}{l}0.304 \\
0.477\end{array}$ & 1.005 & 0.315 \\
\hline $\begin{array}{l}\text { Sexismo benévolo } \\
\text { paternalista }\end{array}$ & $\begin{array}{l}\text { UNSA } \\
\text { UCSP }\end{array}$ & $\begin{array}{l}287 \\
147\end{array}$ & $\begin{array}{l}14.714 \\
12.823\end{array}$ & $\begin{array}{l}4.662 \\
5.216\end{array}$ & $\begin{array}{l}0.275 \\
0.420\end{array}$ & 3.702 & 0.000 \\
\hline $\begin{array}{l}\text { Sexismo dif. } \\
\text { de género }\end{array}$ & $\begin{array}{l}\text { UNSA } \\
\text { UCSP }\end{array}$ & $\begin{array}{l}287 \\
147\end{array}$ & $\begin{array}{l}6.452 \\
6.074\end{array}$ & $\begin{array}{l}2.258 \\
2.760\end{array}$ & $\begin{array}{l}0.133 \\
0.227\end{array}$ & 1.433 & 0.153 \\
\hline $\begin{array}{l}\text { Sexismo } \\
\text { ambivalente }\end{array}$ & $\begin{array}{l}\text { UNSA } \\
\text { UCSP }\end{array}$ & $\begin{array}{l}287 \\
147\end{array}$ & $\begin{array}{l}63.456 \\
58.001\end{array}$ & $\begin{array}{l}17.160 \\
20.241\end{array}$ & $\begin{array}{l}1.012 \\
1.669\end{array}$ & 2.794 & 0.005 \\
\hline
\end{tabular}

En la Tabla 6 se tienen las comparaciones del sexismo en función de la universidad de procedencia de los estudiantes. Se tiene que los estudiantes de la UNSA puntúan más alto en sexismo hostil que sus pares de la UCSP $(\mathrm{p}=0.0 .024)$, al igual que en sexismo benévolo paternalista $(\mathrm{p}=0.000)$, y en sexismo ambivalente como puntaje total $(\mathrm{p}=0.005)$.

\section{DISCUSIÓN}

Históricamente,lasmujereshansidoestigmatizadas de manera negativa, ya sea porque se les ha achacado cierta inferioridad intelectual (Bosch, Manassero, \& Ferrer, 1992) y debilidad moral (Sánchez, 1997), o porque -como continúa ocurriendo hoy en día-, se les relega a roles tradicionales que son socialmente más aceptables, y que son comunicados permanentemente como si se tratase de una realidad perpetua (VelandiaMorales, \& Rincón, 2014).

Lo cierto es que las maneras de entender la masculinidad y la feminidad están cambiando drásticamente en los últimos años (Fernández, Quiroga, del Olmo, \& Rodríguez, 2007; Rabbia, \& Imhoff, 2012). Los varones en la actualidad se preocupan más por su apariencia y recurren a prácticas estéticas, que han sido tradicionalmente usadas por mujeres, aunque sean más frecuentes en los sectores socioeconómicos altos (Villa, 2015). Los varones de hoy, ya no buscan tampoco, mujeres sumisas como pareja o que se ajusten al estereotipo femenino tradicional. De parte de las mujeres, dado que tienen mayor prestigio social, buscan parejas atractivas físicamente, y ya no como ocurría antes, que priorizaban su estatus social (Bastías, Núñez, Avendaño, \& Estrada, 2013).

En ese sentido, las mujeres ya no ven al varón como una figura patriarcal, ya que, al cambiar sus roles de género en diversos ámbitos de la convivencia humana, se han insertado en espacios típicamente masculinos, y con ello, la competencia entre los sexos ha devenido en nuevas formas de sexismo (Tougas et al., 1995). La teoría del sexismo ambivalente, plantea, por ejemplo, que además del sexismo hostil o tradicional, existen formas de sexismo encubiertos que se manifiestan de manera benévola, pero que también implican una actitud prejuiciosa hacia la mujer (Glick, \& Fiske, 1996).

En este estudio, se aplicó el Inventario de Sexismo Ambivalente en una muestra de 434 estudiantes universitarios, con la finalidad de valorar las propiedades psicométricas del instrumento utilizado y analizar las diferencias del sexismo ambivalente entre varones y mujeres, además de considerar como criterio diferencial, la universidad de procedencia y el área de estudios de los universitarios evaluados. En primer lugar, los datos psicométricos, parecen indicar que la prueba tiene validez de criterio y confiabilidad, mientras que la validez de constructo, aunque confirma 
los cuatro factores originales, implica el cambio de algunos ítems de un factor a otro, en relación a la estructura original. Esta situación de la estructura de la prueba, ha sido también reportada por otros estudios realizados con muestras hispanoparlantes (Formiga, 2011; Lemus, Castillo, Moya, Padilla y Ryan, 2008; Vaamonde, \& Omar, 2012).

Por otro lado, los resultados obtenidos sobre el sexismo ambivalente hacia la mujer concuerdan con las investigaciones precedentes donde los niveles de sexismo benevolentes detectados son más altos que el sexismo hostil (Expósito et al., 1998). Una posible explicación de esos puntajes es que la imagen sexista de la mujer en términos benévolos, está más legitimada, incluso entre ellas mismas, como un sistema de actitudes sexistas que sigue considerando a las mujeres de forma estereotipada y limitada a ciertos roles.

Comparativamente, se vio que las mujeres solo tienen puntajes más altos que los varones, en el sexismo benévolo ambivalente de diferencia de género, lo que sugiere que los varones tienen más prejuicios que las mujeres, y que sus ideas son más sexistas, atribuyendo ciertos roles a la mujer. En ese sentido, los varones tienen además de mayores niveles de sexismo hostil, puntuaciones más altas en sexismo benévolo heterosexual, y el sexismo benévolo paternalista; lo que quiere decir que mientras hay varones que asumen un rol de protección hacia la mujer, hay otros que asumen un rol más violento. Estos resultados son similares a los de Cárdenas,Lay, González,Calderón y Alegría (2010), quienes encontraron que los estudiantes universitarios varones de Chile tienen puntajes más altos en sexismo hostil y sexismo benevolente, que sus pares de sexo femenino. En el caso de Cruz, Zempoaltecalt y Correa (2005), encontraron que en los estudiantes mexicanos había un predominio del sexismo hostil en los varones. Esto nos sugiere que habría diferencias culturales en las manifestaciones del sexismo ambivalente, que merecen ser estudiadas con muestras más grandes.

Es importante considerar aquí, que varios estudios han reportado que el sexismo se encuentra significativamente relacionado con la expresión de ira y hostilidad (Garaigordobil, 2015), lo que supone que el sexismo hostil es un factor de riesgo de incurrir en conductas francamente violentas, para quienes obtuvieron puntajes más altos en la dimensión de sexismo hostil. De ahí que sea importante investigar las relaciones entre el sexismo y la violencia.

Al hacer las comparaciones por áreas de estudio, se tiene que existen diferencias significativas en el sexismo benévolo paternalista, de modo que los estudiantes de ingenierías presentan puntajes mayores que los estudiantes de sociales. Esto puede ser un indicador que en un ambiente donde las mujeres son minoría, como en las carreras de ingeniería, podrían ser objeto de sexismo benévolo. Asimismo, tras hacer un análisis comparativo del sexismo en función de la universidad de procedencia, se encontró que los estudiantes de la universidad pública tenían mayores niveles de sexismo hostil, sexismobenévolo paternalista y sexismo ambivalente; que quienes estudian en una universidad privada. Estos resultados pueden hacer hincapié en el hecho de que el sexismo, está mediado por la condición socioeconómica de las personas. Es decir que, a menor estatus socioeconómico, los roles de género se vivencian de manera más tradicional y el sexismo se manifiesta de diversas maneras, tanto de manera hostil como benévola paternalista (Villa, 2015). En ese sentido, algunos estudios indican que efectivamente, en estratos socioeconómicos más bajos existe mayor sexismo y violencia en contra de la mujer (Luna, 2011), lo que nos lleva a relevar el factor social y educativo, para eliminar las diversas formas de sexismo.

Antes de dar fin al presente trabajo, queremos señalar algunas limitaciones que deben superarse en el futuro: en primer lugar, la muestra no ha estado equiparada en términos de cantidad por sexo, carrera o universidad de procedencia; lo que podría afectar los resultados. Por otro lado, es importante que se profundice en las relaciones entre sexismo y conducta violenta, así como con otras variables de estudio, para comprender mejor este fenómeno, que todavía una novedad en nuestro país. Precisamente, por ello, el presente trabajo de investigación, constituye un aporte relevante, porque son pocos los estudios realizados en el país, en materia de neosexismo (Chino y ZegarraValdivia, 2015) o sexismo benevolente (Rottenbacher, 2010). 


\section{REFERENCIAS}

Allport, G. W. (1954). La naturaleza del prejuicio. Buenos Aires: Eudeba.

Barbera, E., \& Martínez-Benlloch, I. (Eds.). (2004). Psicología y género. Madrid: Pearson Prentice Hall.

Bastías, A., Núñez, C., Avendaño, S., \& Estrada, C. (2013). De mujeres y neomujeres: estudio sobre la percepción masculina del atractivo femenino. Salud \& Sociedad, 4(1), 38-46.

Bosch, E., Manassero, M. A., \& Ferrer, V. A. (1992) La misoginia medieval y su repercusión en el concepto de enfermedad mental en la mujer. Revista de Historia de la Psicología, 13(2-3), 329-334.

Baron, R. A. (2005). Psicología. México: Prentice Hall.

Cárdenas, M., Lay, S., González, C., Calderón, C., \& Alegría, I. (2010). Inventario de Sexismo Ambivalente: Adaptación, validación y relación con variables psicosociales. Salud \& Sociedad, l(2), 125-135.

Caycho, T. (2010). Actitudes hacia la homosexualidad masculina y femenina en adolescentes y jóvenes limeños. Revista de Psicología (Universidad César Vallejo), 12, 81-101.

Chino, B. N., \& Zegarra-Valdivia, J. (2015). Neosexismo y autoconcepto en adolescentes peruanos de Educación Básica Regular. Revista de Psicología (Universidad César Vallejo), 17(2), 109-129.

Chirinos, J. L., Brindis, C. D., Salazar, V. C., Bardales, O. T., \& Reátegui, L. R. (1999). Perfil de las estudiantes adolescentes sexualmente activas en colegios secundarios de Lima, Perú. Revista Médica Herediana, 10(1), 49-61.

Crooks, R., \& Baur, K. (2010). Nuestra sexualidad. Mexico: Cengage Learning.

Cruz, C., Zempoaltecalt, V., \& Correa, F. E. (2005). Perfiles de sexismo en la ciudad de México: Validación del cuestionario de medición ambivalente. Revista Enseñanza en Investigación en Psicología, 10(2), 381-385.
Expósito, F., Moya, M., \& Glick, P. (1998). Sexismo ambivalente: medición y correlatos. Revista de Psicología Social, 13(2), 159-169.

Fernández, J., Quiroga, M. A., del Olmo, I., \& Rodríguez, A. (2011). Escalas de masculinidad y feminidad: estado actual de la cuestión. Psicothema, 19(3), 357-365.

Formiga, N. S. (2011). Inventário do sexismo ambivalente em brasileiros: sua acurácia estrutural . Salud \& Sociedad, 2(2), 192-201.

Garaigordobil, M. (2015). Sexismo y expresión de ira: Diferencias de género, cambios con la edad y correlaciones entre ambos constructos. Revista Argentina de Clínica Psicológica, 24(1), 35-42.

Gilbert,L.A.(1999).Reproducing gender in counseling and psychotherapy: Understanding the problem and changing the practice. Applied and Preventive Psychology, 8, 119-127.

Glick, P., \& Fiske, S. (1996). The Ambivalent Sexism Inventory: Differentiating hostile and benevolent sexism. Journal of Personality and Social Psychology, 70, 491-512.

Gotuzzo, H., Peinado, J., Tijero, M., Mayorca, C., \& Badajos, A. (2001). Evaluación de un programa de capacitación en educación sexual y SIDA a docentes del cono norte de Lima-Perú. 1996-1997. Revista Médica Herediana, 12(2), 45-51.

Heilman, M. E. (1995). Sex stereotypes and their effects in the workplace: What we know and what we don't know. Journal of Social Behavior and Personality, 10(6), 3-26.

Lameiras, M., Rodriguez, Y., Carrera, M., \& Faílde, J. (2009). Aproximación conceptual al sexismo ambivalente: Estado de la cuestión. Summa Psicológica, 6(2), 131- 142.

Lemus, S. de, Castillo, M., Moya, M. Padilla, J. L., \& Ryan, E. (2008). Elaboración y validación del Inventario de Sexismo Ambivalente para Adolescentes. International Journal of Clinical and Health Psychology, 8(2), 537-562.

Livia, J., \& Ortiz, M. (2014). Construcción de pruebas psicométricas: Aplicaciones a las ciencias sociales y de la salud. Lima: Universidad Nacional Federico Villarreal. 
Luna, S. E. (2011). Experiencia de masculinidad: la visión de un grupo de hombres guatemaltecos. Salud \& Sociedad, 2(3), 250-266.

Mingo, A., \& Moreno, H. (2015). El ocioso intento de tapar el sol con un dedo: violencia de género en la universidad. Perfiles Educativos, 37(148), 138155.

Moya, M. C. (1990). Favoritismo endogrupal y discriminación exogrupal en las percepciones de las características sexoestereotipadas. En G. Musitu (Comp.), Procesos Psicosociales Básicos (pp. 221-228). Barcelona: PPU.

Moya, M. y Expósito, F. (2001). Nuevas formas, viejos intereses. Neosexismo en varones españoles. Psicothema, 13(4), 643-649.

Moya, M., Páez, D., Glick, P., Fernández, I., \& Poeschl, G. (2002). Sexismo, masculinidadfeminidad y factores culturales. Revista Española de Motivación y Emoción, 3, 127-142.

Portilla, Ch. \& Vilches, F. (2007). Homofobia en estudiantes universitarios. Revista de Psicología (Universidad Católica de Santa María), 4, 48-63.

Rabbia, H. H., \& Imhoff, D. (2012). Concepciones sobre heterosexualidad y actitudes hacia la disidencia sexual en estudiantes de Psicología de Córdoba. Revista Argentina de Ciencias del Comportamiento, 4(3), 22-29.
Rottenbacher, J. M. (2010). Sexismo ambivalente, paternalismo masculino e ideología política en adultos jóvenes de la ciudad de Lima. Pensamiento Psicológico, 14, 9-18.

Rudman, L., \& Glick, P. (2001). Prescriptive gender sterotypes and backlash toward agentic woman. Journal of Social Issues, 57(4), 743-762.

Sánchez, T. (1997) Crónica y vida de las mujeres del siglo XVI (a partir de fuentes primarias y tratados morales). Revista de Historia de la Psicología, 18(1-2), 343-354.

Tougas, F., Brown, R., Beaton, A. M., \& Joly, S. (1995). Neosexism: Plus Ça Change, Plus C'est Pareil. Personality and Social Psychology Bulletin, 21(8), 842-849.

Vaamonde, J. D., \& Omar, A. (2012). Validación argentina del Inventario de Sexismo Ambivalente. Alternativas en Psicología, 16(26), 47-58.

Velandia-Morales, A., \& Rincón, J. C. (2014). Estereotipos y roles de género utilizados en la publicidad transmitida a través de televisión. Universitas Psychologica, 13(2), 517-527.

Villa, J. (2015) Cuerpo, masculinidad y estilo en jóvenes de sectores altos de Lima. Debates en Sociología, 40, 61-91.

Fecha de recepción: 24 de junio, 2016

Fecha de aceptación: 4 de julio, 2017 